Method Databases including conference abstracts and grey literature were searched using pre-defined terms. Screening of title and abstract, followed by full text review against inclusion criteria. Two reviewers extracted data from included studies and assessed Hayden risk of bias. Findings were subjected to narrative synthesis as meta-analysis was not possible.

Results 29306 records were screened resulting in 37 included studies. There was a plethora of prognostic variables studied, including demographic, laboratory, electrocardiography, echocardiogram and clinical variables as well as studies that developed models from multiple variables. The primary variables were often only studied once, but even for the few that were investigated more than once, were adjusted for by different covariates and so could not be combined.

Conclusions This systematic review failed to find clinically useful prognostic variables to identify patients in the last year of life. Although we identified a variety of proposed prognostic variables, these were explored in a single or in only a few studies, often in restricted populations meaning they cannot be generalised. Identifying good predictors of this phase of life is useful to facilitate advance care planning. Future prognostication research should concentrate on variables measured over time and studying real life populations more representative of patients with advanced heart failure and comorbidity.

\section{IMPROVING COMPLETION RATES OF DISCHARGE SUMMARIES FOR PATIENTS THAT DIE DURING THEIR ADMISSION TO HOSPITAL}

Heather Y Holyoak, Ningyu Chai, Farid Bazari. Kingston Hospital NHS Foundation Trust

10.1136/spcare-2020-PCC.84

Introduction All patients admitted to hospital should have a discharge summary completed, including those that die during their admission. This ensures good continuity of care between secondary and primary care and allows for Bereavement support to be offered. We looked at the current level of discharge summaries being completed for these patients, and the quality of information provided.

Methods We initially identified patients who died between November and December 2018. 114 patients died. Only 35 (31\%) patients had a discharge summary completed. A survey of doctors highlighted confusion about what documentation was required, they felt that they are often too busy to do summaries and see deceased patients as a 'lesser priority'.

A project was conducted to highlight the importance of these summaries and to improve the quantity and quality of them. This included presenting our initial data at Grand Round, the Junior Doctor Forum and piloting a 'Bereavement Box' on one of the elderly care wards. This was used in morning board rounds to highlight summaries that needed to be done, but also as an opportunity to debrief and learn from deaths.

Results Following these changes, we looked at the deaths in June 2019. 55 patients died. $33(60 \%)$ patients had a discharge summary completed. $100 \%$ of patients who died on the ward with the 'Bereavement Box' had a discharge summary.

Conclusions With these interventions, there was a $100 \%$ increase in discharge summaries being completed. However, there are still further improvements to be made. We plan to roll out 'Bereavement boxes' to all wards, make an automatic notification sent to the GP upon a patient's death and introduce Medical Examiners within the Trust. We hope that these interventions will ensure that all patients will have discharge summaries completed and there will be improved continuity of end of life care.

\section{THE CHALLENGE OF DE-ESCALATING CARE; TRANSFER FROM ICU TO A WARD SETTING FOR END OF LIFE CARE}

Sinead Hutcheson, Kiran Kaur, Clare Lenaghan, Pamela Oakes, Brenda Mcllroy, Natalie McCleary, Jenny Rankin. Belfast Health and Social Care Trust

\subsection{6/spcare-2020-PCC.85}

Background The Palliative Care Team (PCT) in an acute hospital are referred patients from the regional Intensive Care Unit (ICU) who have had withdrawal of active treatment and are being discharged to a ward for end of life care (EOLC). Transfer between teams and wards is a challenging time. ICU patients are often on intravenous (IV) infusions of drugs which require conversion to the subcutaneous (SC) route.

Aim The aim of this review is to assess the care pathway and symptom control of patients in ICU who are recognized to be dying, who are being transferred from ICU to a ward for EOLC.

Methods Retrospective audit. PCT database was searched over a 12 month period to identify patients. Literature search and data collection completed.

Results 9 patients were eligible for inclusion. 3 patients were referred to PCT but not seen as they died on day of referral. All patients with PCT input in ICU had a continuous subcutaneous infusion (CSCI) commenced prior to discharge, and were well symptom controlled for remainder of admission. One patient was identified as having uncontrolled symptoms; they had been discharged from ICU on a weekend and had no PCT input. 3 patients had IV infusions of opioid and/or midazolam converted to CSCI with a $40-50 \%$ dose reduction which maintained symptom control.

Conclusions This review highlights that early PCT input into ICU discharges for EOLC facilitates better symptom control. Out of hours transfers are highlighted as a time of risk. The IV to SC conversions used here were tolerated well, however a $50 \%$ dose reduction is not seen as standard as the management of this conversion will vary depending on the prescriber's assessment of the patient. Further review of this care pathway and prescribing practice is merited to allow development of guidance.

\section{DIGNIFIED DEATH: THE IMPACT OF PALLIATIVE CARE ON POLYPHARMACY AND ANTICIPATORY PRESCRIBING}

Yvette Jaffe, Emily Ward, Alan Poots, Victor Jaffe, Ruth Caulkin. Imperial College School of Medicine, Chelsea and Westminster Hospital, Royal Free NHS Foundation Trust

\subsection{6/spcare-2020-PCC.86}

Background and Aims At the end of life, a person's needs and goals change. It is, therefore, important to review medications to ensure optimum symptom control and to deprescribe 